\title{
PREDIÇÃO DA ESTRUTURA DIAMÉTRICA DE ESPÉCIES COMERCIAIS DE TERRA FIRME DA AMAZÔNIA POR MEIO DE MATRIZ DE TRANSIÇÃO
}

\author{
PREDICTION OF THE DIAMETRIC STRUCTURE OF COMMERCIAL TREE SPECIES OF THE \\ AMAZONIAN UPLAND FOREST, USING TRANSITION MATRIX
}

\author{
Ulisses Silva da Cunha ${ }^{1}$ Sebastião do Amaral Machado ${ }^{2}$ Afonso Figueiredo Filho ${ }^{3}$ \\ Carlos Roberto Sanquetta ${ }^{4}$
}

\section{RESUMO}

Esta pesquisa se originou de uma base de dados de dois inventários a 100\% realizados em 1984 e 2000 sobre uma mesma área de 576 ha de floresta tropical primária, localizada na Floresta Nacional do Tapajós, Belterra, Pará. A pesquisa objetivou avaliar a evolução da estrutura diamétrica de seis espécies comerciais de terra firme da Amazônia no período 1984-2000 e projetá-la para 2016, utilizando-se de matriz de transição. Nos dois inventários, as CAP foram medidas com fita métrica, sendo que em 1984, as alturas comerciais foram medidas com o hipsômetro de Weiss e, em 2000, estimadas com o uso de varas; o DAP mínimo em 1984 foi de $55 \mathrm{~cm}$ para todas as espécies e, em 2000, foi adotado o DAP mínimo de $35 \mathrm{~cm}$. A construção da matriz de transição foi precedida de análise exploratória de dados (AED), em que se utilizou o box plot (caixa-de-bigodes) na detecção de outliers (observações discrepantes) e o gráfico stem-and-leaf (tronco-e-folhas) para filtrar as observações extremas. A análise da estrutura diamétrica mostrou que a evolução do DAP, observada no período 1984-2000, apresentou comportamento diferenciado por espécie, embora mantendo a forma básica da distribuição típica de cada espécie, enquanto a projeção da estrutura diamétrica para 2016, usando matriz de transição, manteve-se com a tendência esperada, com base nas taxas de crescimento expressas pelas raízes latentes da matriz de transição o que demonstra estar compatível com o perfil da estrutura diamétrica observada em 2000.

Palavras-chave: taxa de crescimento, floresta tropical, distribui ção diamétrica.

\begin{abstract}
This research was originated from a data base of two 100\% inventories accomplished in 1984 and 2000 on a same area of 576 hectares of primary tropical forest, located in the National Forest of Tapajós, Belterra, Pará, Brazil.The objective of this research was to evaluate the evolution of diameter structure of 6 commercial tree species in an Amazonian upland forest for the period from 1984 to 2000, and to project it to the year 2016, by using transition matrix. In the two inventories, circunference at breast height (CBH), in $\mathrm{cm}$, were measured with measuring tape, and in 1984, the commercial heights were measu red with the hypsometer of Weiss and, in 2000, estimated with the use of sticks; minimum DBH in 1984 were $55 \mathrm{~cm}$ for all species and, in 2000, minimum DBH of $35 \mathrm{~cm}$. The construction of the transition matrix was preceded by an exploratory data analysis (EDA), where the box plot was used for outliers detection and the graph stemand-leaf was used to filter the extreme observations. The analysis of the diametric structure showed that the evolution of DBH observed in the period 1984-2000, presented behavior differentiated by species, although maintaining the basic form of the typical distribution of each species, while the projection of the diametric structure for 2016, using transition matrix remained with the expected tendency, according to the expressed growth rates for the latent roots of the transition matrix, demonstrating to be compatible with the profile of the diametric structure observed in the year 2000.
\end{abstract}

Key words: growth rate, tropical forest, diametric distribution.

1. Engenheiro Florestal, Dr., Professor do Curso de Engenharia Florestal, Universidade do Amazonas, Av. Gal. Rodrigo Otávio J. Ramos, 3000, Aleixo, Mini-campus, CEP 69077-000, Manaus (AM). ucunha@fua.br

2. Engenheiro Florestal, Dr., Professor Sênior do Curso de Engenharia Florestal, Universidade Federal do Paraná, Rua Lothário Meissner, 340, Jardim Botânico, Campus II, CEP 80210-170, Curitiba (PR). sammac@floresta.ufpr.br PQ $1 \mathrm{~A}-\mathrm{CNPq}$.

3. Engenheiro Florestal, Dr., Professor Sênior do Curso de Engenharia Florestal, Universidade Federal do Paraná, Rua Lothário Meissner, 340, Jardim Botânico, Campus II, CEP 80210-170, Curitiba (PR). afig@ floresta.ufpr.br - PQ 2B - CNPq.

4. Engenheiro Florestal, Dr., Professor do Curso de Engenharia Florestal, Universidade Federal do Paraná, Rua Lothário Meissner, 340, Jardim Botânico, Campus II, CEP 80210-170, Curitiba (PR). sanqueta@floresta.ufpr.br PQ 2B - CNPq. 


\section{INTRODUCÃO}

A Floresta Nacional do Tapajós reúne um dos maiores acervos em pesquisas florestais da Amazônia, seja de natureza científica ou técnica. Mesmo considerando os avanços da pesquisa florestal na Amazônia durante a última década, vários componentes do ecossistema ainda necessitam ser investigados. Como exemplo, pode-se citar a falta de um melhor conhecimento sobre dinâmica florestal a qual depende de um período mais longo de observação, quase sempre exigindo o estabelecimento de parcelas permanentes.

Apesar de serem mais eficientes para estudos de dinâmica e mudanças estruturais em uma floresta, nem sempre existem dados disponíveis de parcelas permanentes com a qualidade exigida para o desenvolvimento de modelos, pois, em muitos casos, existem problemas na condução e acompanhamento das medições, perda de dados, abandono total ou parcial ou até eventuais distúrbios naturais sobre determinadas parcelas, dificultando o controle e a recupera ção de dados.

A busca de fontes alternativas de obtenção e geração de dados para análise deve ser um desafio constante dos pesquisadores. Nesse sentido, considerando que normalmente os invent ários a $100 \% \mathrm{em}$ escala comercial abrangem áreas em módulos de 50, 96, 100 ou mais hectares, a disponibilidade de dados, em áreas desse porte, é um aspecto positivo em termos de potencial de pesquisas.

Em florestas tropicais, o crescimento da floresta ao longo de um período de anos tem três componentes: i) incremento individual da árvore; ii) mortalidade e, iii) ingresso ou aparecimento de novas árvores em tamanho mensurável das classes de DAP em função da regeneração (Adlard, 1990).

O conhecimento da regeneração e do incremento é essencial em termos de projeção da produção em longo prazo. Dependendo da taxa de crescimento e do status competitivo das espécies, é possível para alguns indivíduos da floresta crescerem até $70 \mathrm{~cm}$ com base na semente em 30 anos, ou seja, a regeneração pode afetar significativamente a produção dentro do ciclo de corte, trazendo implicações para o manejo florestal (Alder e Synnott, 1992). Apesar disso, aspectos como mortalidade, regeneração, recrutamento e aproveitamento não puderam ser abordados neste estudo, pela ausência dessas informações proporcionadas pelos inventários, restringindo o escopo da presente análise aos aspectos relacionados com o crescimento e a produção.

Os modelos matriciais de crescimento têm suas raízes no modelo proposto por Leslie (1945), delineado para investigar o efeito da estrutura de idades nas taxas de crescimento de popula ções de animais. Usher (1966) é considerado um dos pioneiros na adaptação de matriz markoviana pura no desenvolvimento de modelos de distribuição diamétrica. A partir daí, foram sendo implementadas várias aplicações na área florestal, como Wadsworth (1977), um dos primeiros a fazer uso do modelo de Usher para predizer o crescimento de florestas tropicais.

No modelo de Usher, cada classe diamétrica tem uma ou mais taxas de transição associadas, respectivamente, com: i) a proporção de árvores que permaneceram na mesma classe após um período de crescimento $\mathrm{p}$; ii) a proporção de árvores que se moveram para classes imediatamente superiores no

período $\mathrm{p}$; iii) a proporção das árvores que morreram no período $\mathrm{p}$ e, iv) proporção de árvores recrutas (ingresso) no período $\mathrm{p}$.

Segundo Higuchi (1987), o uso da cadeia de Markov é uma alternativa para os estudos de crescimento de florestas naturais, uma vez que, ao contrário dos modelos clássicos usados em plantações florestais, não se apóia em variáveis de difícil acesso em florestas naturais tais como a idade e o índice de sítio.

Assim, com base em tal conjunto de taxas de transição para cada classe de diâmetro sobre a distribuição diamétrica presente, a distribuição do próximo período pode ser projetada, assumindo-se que as taxas de mudanças atuais estão em equilíbrio e que serão as mesmas no futuro.

Segundo Adlard (1990), o conhecimento das taxas de crescimento das espécies seja em uma floresta natural ou plantada é a base para o seu manejo. A taxa de crescimento e sobrevivência de árvores é fortemente influenciada pelas variáveis luz, água e nutrientes. A produção máxima de uma floresta tropical depende do tipo de floresta e dos fatores de sítio, podendo-se estabelecer uma área basal máxima de 44-55 $\mathrm{m}^{2} /$ ha para essas florestas (Assmann, 1970; Alder, 1990). 
De acordo com Alder (1995), em florestas tropicais, o incremento diamétrico pode ser predito empiricamente com base no diâmetro da árvore ou área basal, nível de competição e variáveis de sítios. Outras variáveis de importância relativa como idade, índice de sítio e até altura comercial não são fáceis de serem mensuradas em florestas tropicais o que as torna um fator limitante na constru ção de modelos de interesse para o manejo florestal. Em virtude dessas e outras dificuldades freqüentes em modelos clássicos, o uso de matriz de transição torna-se uma alternativa para estudos de crescimento em florestas naturais em função das variáveis de fácil acesso que podem ser incorporadas aos modelos básicos construídos.

De acordo com NIST (2001), a análise exploratória de dados (AED) emprega uma variedade de técnicas gráficas para: 1) maximizar o conhecimento sobre um conjunto de dados; 2) investigar hipóteses sobre a estrutura dos dados; 3) detectar outliers; 4) examinar relações entre variáveis; 5) analisar aspectos distributivos.

No entanto, os métodos gráficos nem sempre são uma solução efetiva nem um substituto dos métodos analíticos convencionais, pois, segundo Ellison (1993), a AED é mais apropriada, quando existe grande variabilidade nos dados e quando os efeitos dos tratamentos não são imediatamente aparentes.

Esta pesquisa objetivou avaliar a evolução da estrutura diamétrica de seis espécies comerciais de terra firme da Amazônia no período 1984-2000 e projetá-la para 2016, utilizando-se matriz de transição.

\section{MATERIAL E MÉTODOS}

A área utilizada na presente pesquisa com destaque na (Figura 1), é quadrada com 2,4 km de lado, tem 576 ha, compõe-se de seis blocos contíguos de 96 ha de floresta primária, cada um medindo $800 \mathrm{~m}$ no sentido leste-oeste e $1.200 \mathrm{~m}$ no sentido norte-sul e faz parte da Floresta Nacional do Tapajós (FLONA Tapajós) que tem 600.000 ha e está situada entre os paralelos de $2^{\circ} 40^{\prime}$ a $4^{\circ} 10^{\prime}$ de latitude sul e os meridianos de $54^{\circ} 45^{\prime}$ a $55^{\circ} 30^{\prime}$ de longitude oeste de Greenwich, município de Belterra, Pará. O seu manejo está previsto para 2001, como parte do programa a ser executado pela Treviso Agropecu ária Ltda, mediante Convênio de Cooperação entre o Instituto Brasileiro do Meio Ambiente e dos Recursos Naturais Renováveis (IBAMA) e a International Tropical Timber Organization (ITTO), em andamento desde 1999.

De acordo com RADAMBRASIL (1976), predominam, na área, os latossolos amarelos distróficos com diferentes texturas, profundos, ácidos, friáveis, onde ocorre a floresta densa. Como variação, tem-se também o latossolo amarelo distrófico plíntico de textura média e argilosa, os solos concrecionários lateríticos indiscriminados distróficos e areias quartzosas distróficas. Esses solos ocorrem em diversos tipos de relevo, variando desde plano a forte ondulado, sob vegetação de floresta densa e são originados com base em sedimentos do Terciário.

A Floresta Ombrófila Densa subdividida pelos quadrantes ecológicos (litologia, morfologia e clima), em termos de aspectos fisionômicos e florísticos, caracteriza a tipologia florestal dominante na região. Apresenta-se também na área uma fisionomia florestal densa, com indivíduos emergentes, ocupando as áreas aplainadas dos platôs do Xingu-Tapajós, caracterizada não só por apresentar alto volume, como também pela presença de espécies de alta cotação de preços nos mercados externo e interno (IBAMA, 1997).

\section{Base de dados}

Nesta pesquisa, com base em informações de dois inventários distintos sobre uma mesma área de 576 ha, desenvolveu-se uma metodologia de recuperação, análise e tratamento estatístico desses dados visando a avaliar a produção presente e predizer a produção futura com margem aceitável de erro.

Os dados são provenientes de duas fontes distintas, mas sobre a mesma área: 1) Inventário a 100\% (FUPEF, 1984) e, 2) Inventário a 100\% (Treviso, 2000). Em 1984, as equipes da FUPEF percorreram as linhas de inventário distribuídas a cada $100 \mathrm{~m}$, cobrindo sempre o lado de uma das faixas de $50 \mathrm{~m}$ dispostas no sentido norte-sul. Em 2000, as equipes da Treviso percorreram as linhas de inventário distribuídas a cada $50 \mathrm{~m}$, com o caminhamento simultâneo de duas equipes por linha, recobrindo duas faixas laterais internas de $25 \mathrm{~m}$, ora se deslocando para dentro e para fora da linha-base situada em posição frontal às linhas de inventário. 
Os dois inventários foram previamente processados e, com base nas coordenadas de locação das árvores $\left(\mathrm{x}_{\mathrm{i}}, \mathrm{y}_{\mathrm{i}}\right)$, elaboram-se dois tipos de mapas por espécie, objetivando cruzar informações que permitissem recuperar, por diferença diamétrica, numeração seqüencial e propriedade de vizinhança, a mesma árvore abordada pelos dois inventários. O primeiro contendo informações sobre o diâmetro à altura do peito (DAP), em cm, de cada indivíduo e, o segundo contendo a numeração atribuída pelas equipes em cada inventário.

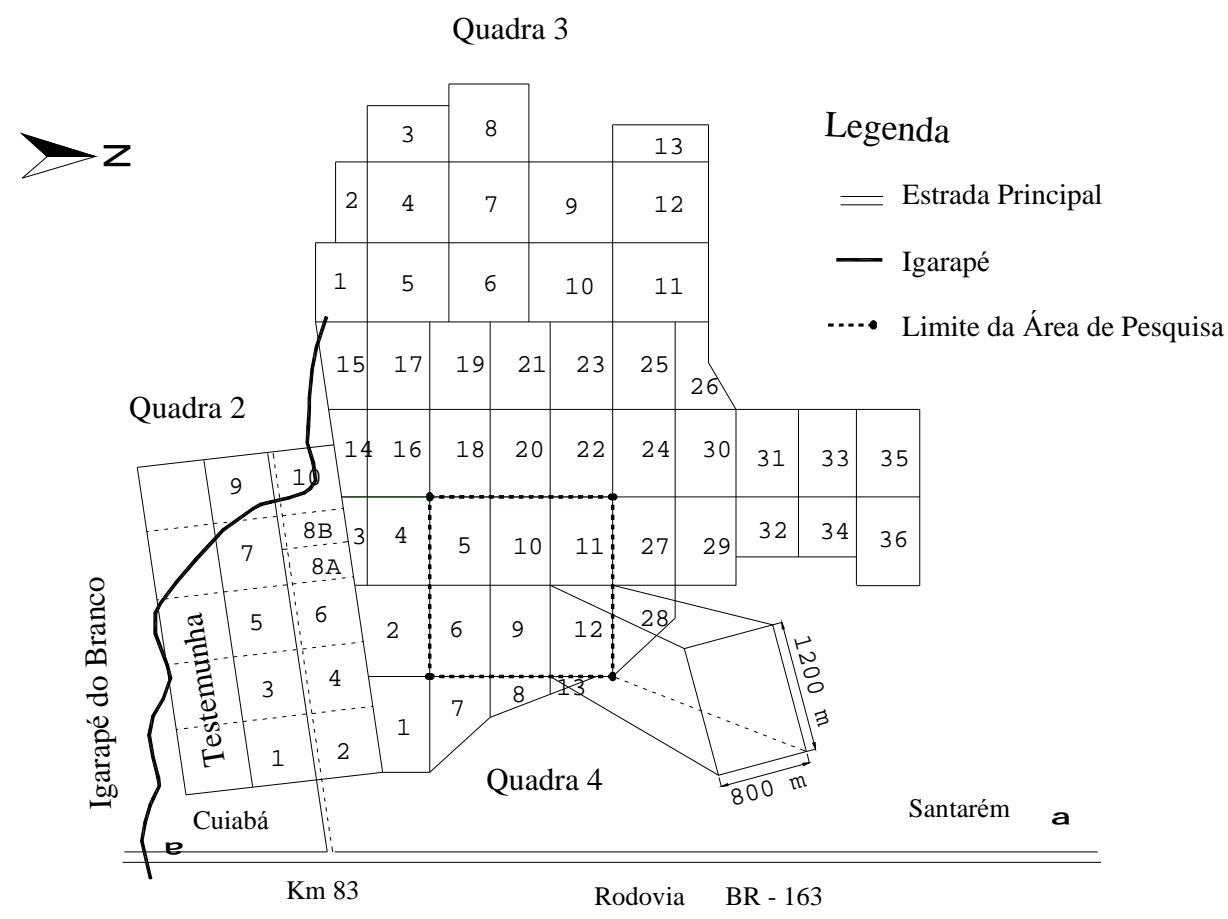

FIGURA 1: Localização e delimitação da área de pesquisa. Fonte: FUPEF (1984); IBAMA (1997).

Inicialmente, procurou-se identificar e marcar os pares de árvores mais próximas, observando-se e comparando-se os DAP medidos nas duas ocasiões. Em seguida, os pares marcados foram transferidos para o segundo mapa, contendo a numeração dos dois inventários. O passo seguinte consistiu na ordenação dos dados árvore-a-árvore, de acordo com a respectiva numera ção em cada ocasião, visando a permitir visualizar o comportamento das mesmas variáveis durante o período 1984-2000.

Nos dois inventários, as circunferências à altura do peito (CAP) foram medidas com fita métrica. As alturas comerciais em 1984, foram medidas com o hipsômetro de Weiss e, em 2000, estimadas visualmente com o uso de varas; o DAP mínimo, em 1984, foi de $55 \mathrm{~cm}$ e em 2000, adotou-se o DAP mínimo de $35 \mathrm{~cm}$. Em 1984 foram inventariados $100 \%$ dos indivíduos com $\mathrm{DAP} \geq 55 \mathrm{~cm}$ o que permitiu que 210 espécies fossem identificadas. Em 2000, foram inventariados 100\% dos indivíduos com DAP $\geq 35 \mathrm{~cm}$, à exceção de trinta espécies estabelecidas num acordo técnico entre IBAMA/Treviso, dentre as quais se destacam Envirapreta (Gatteria poeppigiana Mart.), Tachi preto (Tachigalia paniculata Aubl.), etc.

Segundo Silva (2000), a identificação dos inventários florestais, baseados apenas em nomes populares, pode causar confusão e até erros irreparáveis, pois, geralmente, são denominações que variam de uma região para outra e até dentro da mesma região, devendo merecer atenção especial por parte de quem as utiliza, principalmente em processos de comercialização. Apesar do trabalho adicional de identificação de parte do material botânico em ambos os inventários por herbários especializados, considerou-se que este não seria suficiente para assegurar uma identificação sem falhas, razão pela qual se preferiu restringir a an álise a poucas espécies cujas características são de fácil domínio por mateiros da região.

Para as análises, foram escolhidas as seguintes espécies: 1) Minquartia macrophylla Ducke 
(Acariquara); 2) Carapa guianensis Aubl. (Andiroba); 3) Bertholletia excelsa Humb e Bonpl. (Castanheira); 4) Goupia glabra Aubl. (Cupiúba); 5) Manilkara huberi Ducke (Maçaranduba); 6) Bagassa guianensis Aubl. (Tatajuba). A lista é composta por espécies de importância comercial aparentemente sem-problemas de identificação, conforme relatado por (EMBRAPA, 2000). O principal critério de escolha baseou-se na facilidade de identificação por mateiros da região, tendo sido considerado que, nas duas ocasiões, diferentes mateiros não tiveram problemas na identificação.

Preferiu-se utilizar a mediana do $\operatorname{DAP}\left(\mathrm{d}_{1}, \mathrm{~L} \mathrm{~d} \mathrm{~d}_{\text {med }}, \mathrm{L} \mathrm{d}_{\mathrm{n}}\right)$ e da área transversal $\mathrm{g}\left(\mathrm{g}_{1}, \mathrm{~L} \mathrm{~g}_{\text {med }}, \mathrm{L} \mathrm{g}_{\mathrm{n}}\right)$, em vez da média aritmética nas análises de crescimento, com base nas seguintes justificativas demonstradas por Mosteller e Tukey (1977): i) o uso da média aritmética deve ser precedido de análise exploratória, quando o tipo de dados apresentar outliers ou quando por conveniência, for requerida a linearidade de um modelo de regressão; ii) a média aritmética pode apresentar estimativas com tendências para dados que não seguem uma distribuição normal e, iii) por ser comprovadamente o protótipo de uma medida resistente, a mediana é usada com alta eficiência na maioria das situações de análise exploratória e quando os dados não seguem uma distribuição normal o que reflete o caso das variáveis de crescimento em florestas tropicais. A opção pelo uso da mediana, em sentido técnico, deveu-se a impossibilidade de sua determinação em termos de observações individuais o que exigiu que a área de estudo fosse dividida em módulos.

\section{Valores discrepantes (Outliers)}

Barnett e Lewis (1995) definem outlier em um conjunto de dados como sendo uma observação que parece ser inconsistente com o conjunto de dados remanescentes. outliers podem indicar várias coisas importantes sobre um modelo: i) modelo incompatível com os dados; ii) omissão de variáveis importantes. Quaisquer outliers são sempre valores extremos ou relativamente extremos em uma amostra. Para Draper e Smith (1981), rejeição automática de outliers não é um procedimento correto e as regras propostas para rejeição de outliers devem incluir a reanálise sem essas observações as quais, dependendo das circunstâncias, podem ser portadoras de informações vitais dos indivíduos de uma população.

Na rejeição de outliers, teve-se a precaução em diagnosticar os verdadeiros outliers, diferenciandoos das observações influentes como os breakdown points e leverage points. Gnanadesikan (1997) define breakdown point como a maior fração das observações em uma amostra que podem ser valores extremos sem distorcer o valor do estimador. Statgraphics (1999) define leverage point como a estatística que mede o quanto cada coeficiente estimado poderá mudar se cada observação for removida dos dados. Diz-se que um ponto tem influência significativa quando o valor do seu leverage for maior do que três vezes o número de parâmetros dividido pelo número de observações. Adotou-se também o critério preconizado por Barnett e Lewis (1995) o quais consideram que nem toda observação extrema é um outlier e que o efeito de um outlier é sua influência sobre a estimativa dos parâmetros no modelo.

\section{Análise exploratória de dados (AED)}

A AED é necessária antes de se explorar adequadamente a natureza dos dados e suas propriedades, sendo um complemento de suma importância que deve anteceder o uso de certas ferramentas estatísticas como regressão, evitando-se erros grosseiros e a análise parcial com a geração de resultados duvidosos. Fez-se a análise de dados por processo de monitoramento visando a revelar outliers mediante técnicas gráficas como stem-and-leaf e box plot.

O box plot é uma excelente ferramenta por permitir visualizar a locação, a dispersão, a simetria, o peso das caudas e os outliers independente da forma da distribuição de um conjunto de dados. Além disso, o box plot é construído com base na mediana e nos quartos associados à coleção de dados o que o torna resistente a valores perturbadores dentro das barreiras de outliers e atraentes em análise exploratória de dados (Hoaglin et al. 1992).

O stem-and-leaf é usado para organizar e sumarizar valores numéricos produzindo uma figura compacta das propriedades de um conjunto de dados sem-teoria elaborada. Por regra, qualquer ponto que exceder a dois intervalos interquartis (ex. 0,25 a 0,75 ), valendo-se do quartil mais próximo, é considerado 
um valor extremo.

\section{Modelo de crescimento com matriz de transição}

A palavra estocástico deriva do grego e significa aleatório ou chance. $\mathrm{O}$ antônimo é determinístico ou certeza. Um modelo determinístico prediz um simples resultado proveniente de um conjunto de circunstâncias. Um modelo estocástico prediz um conjunto de resultados possíveis com base em probabilidades. Processos estocásticos são caracterizados pelas relações de dependência que existe entre suas variáveis. Taylor e Karlin (1998) definem processo estocástico como uma família de variáveis aleatórias $\mathrm{T}_{\mathrm{t}}$, podendo o índice $\mathrm{t}$, corresponder à unidade de tempo.

De acordo com Taylor e Karlin (1998), um processo de Markov $\left\{\mathrm{T}_{\mathrm{t}}\right\}$ é um processo estocástico com a propriedade de que dado o valor de $T_{t}$, os valores de $T_{t+2 p}$ não são influenciados pelos valores de $\mathrm{T}_{\mathrm{t}+\mathrm{p}}$. Em outras palavras, a probabilidade de qualquer comportamento particular futuro do processo, quando seu estado corrente é exatamente conhecido, não é alterado por conhecimento adicional concernente com seu comportamento passado. Quando as probabilidades de transição da primeira etapa são independentes da variável tempo $\mathrm{t}$, diz-se que a cadeia de Markov tem probabilidades de transição estacionária.

Para que as projeções feitas com matrizes de transição sejam válidas, é necessário que uma condição básica seja satisfeita: que as taxas de transição sejam homogêneas, isto é, que as taxas de transição permaneçam constante durante o período sobre o qual a projeção é feita. Considerando-se que a análise será feita enfocando aspectos evolutivos, assume-se que as taxas de ingresso e mortalidade são constantes e iguais a zero.

É difícil estabelecer que não haverá mudanças nas probabilidades de transição no tempo, especialmente nos casos de populações de árvores que vivem longos períodos. A dificuldade em testar a homogeneidade quase sempre se deve à falta de dados observados em períodos mais longos. Na presente pesquisa, o período de observação (1984-2000) é de 16 anos, característica essa que a destaca como um dos poucos estudos com um tempo de observação superior a 10 anos na Amazônia, mas apresenta, como principal limitação, a ausência de medições em períodos anteriores a 2000.

Em um modelo de matriz de transição para prognose da abundância por espécie, tem-se a abundância inicial no momento $\mathrm{t}$ (presente) e pretende-se projetar a abundância para um momento $t+2 p$ (futuro). Mas, conforme demonstrado por Sanquetta et al. (1997), para se obter a prognose, faz- -se necessário recorrer ao cálculo das probabilidades das possíveis combinações do processo de crescimento por classe diamétrica, podendo resultar numa matriz de transição $\mathrm{T}$ associada à seguinte expressão:

$$
\begin{aligned}
& \begin{array}{llcccc}
\text { Classes } & 1 & 2 \mathrm{~L} & \mathrm{~L} \mathrm{~L} & \mathrm{k} & \mathrm{k}+\mathrm{m} \\
\text { DAP } & & \text { Estado inicial no tempo } t &
\end{array}
\end{aligned}
$$

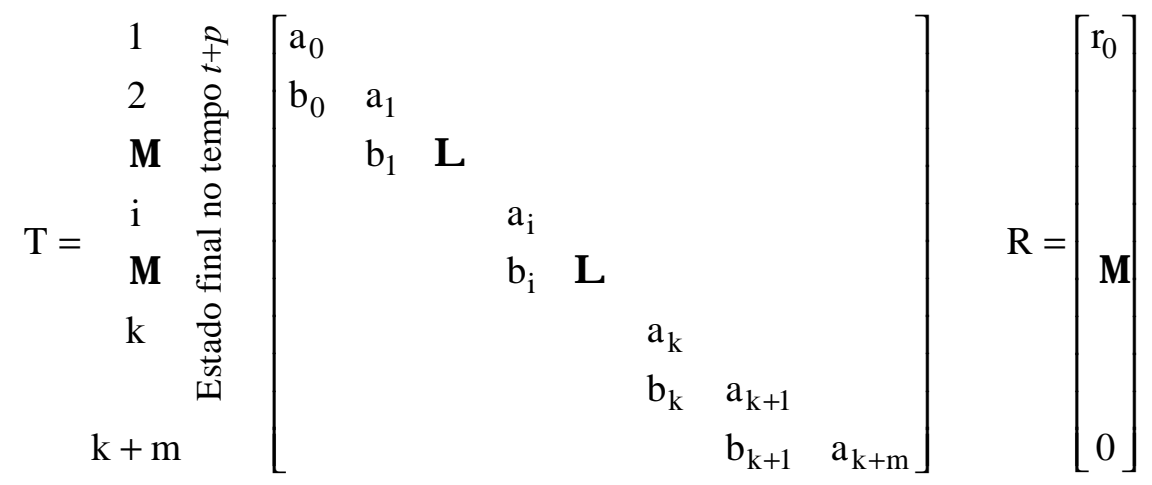

Sendo: $\mathrm{T}=$ transposta da matriz probabilística de transição da floresta para o período $\mathrm{p} ; \mathrm{R}=$ vetor derecrutamento (ingresso) na floresta durante o período $\mathrm{p} ; \mathrm{r}_{0}=$ representa a taxa de recrutamento constante 
na menor classe diamétrica; $\mathrm{a}_{\mathrm{i}}=$ ocupa posições na diagonal principal como a probabilidade de que uma certa árvore de uma determinada classe de diâmetro permaneça na mesma classe de diâmetro i durante o período $\mathrm{p} ; \mathrm{b}_{\mathrm{i}}=$ ocupa posições na subdiagonal como a probabilidade de que os indivíduos de uma classe de diâmetro i mudem para a classe imediatamente superior durante o período $\mathrm{p} ; \mathrm{k}=$ número de classes de diâmetro do vetor do número de árvores; $\mathrm{m}$ = número de classes de diâmetros adicionadas durante o período de transição $\mathrm{p}$.

A matriz de transição $\mathrm{T}$ é quadrada e seus elementos são as probabilidades de transição movendo-se do estado inicial $(\mathrm{t})$ para o estado final $(\mathrm{t}+\mathrm{p})$ no período $\mathrm{p}$. A diagonal principal da esquerda para a direita fornece a proporção de indivíduos que não saíram da sua classe no período $\mathrm{p}$. A primeira subdiagonal fornece a proporção de indivíduos que se moveram para as classes seguintes, sendo que a probabilidade de haver transição na última classe é zero.

As principais propriedades do modelo matricial são que as combinações lineares resultantes assumem que as probabilidades de transição permanecem constantes no período de projeção. Além disso, o modelo de USHER explicitado na equação (1), deve atender à condição $A_{i}+B_{i}=1$, exceto para $a_{n}$ que pode ser igual a 1 . O que significa que todas as árvores, em determinada classe, têm a chance de permanecer ou se mover para um ou mais classes superiores.

Para cada espécie com $\mathrm{k}$, classes diamétricas, cada uma com $\mathrm{n}_{\mathrm{i}}=1,2, \mathrm{~L}, \mathrm{k}$ indivíduos, o tamanho dessa população será dado pelo vetor coluna $\mathrm{N}_{\mathrm{t}}$. A projeção do vetor $\mathrm{N}_{\mathrm{t}+\mathrm{p}}$ é obtida multiplicando-se a matriz de transição $\mathrm{T}$ pelo vetor inicial $\mathrm{N}_{\mathrm{t}}$, ilustrado pela equação (2) . Para se obter a projeção do vetor $\mathrm{N}_{\mathrm{t}+2 \mathrm{p}}$ deve-se multiplicar a matriz potência $\mathrm{T}^{2}$ pelo vetor inicial $\mathrm{N}_{\mathrm{t}}$, de acordo com a equação (3).

$$
\begin{aligned}
& \mathrm{N}_{\mathrm{t}+\mathrm{p}}=\mathrm{T} \times\left[\begin{array}{c}
\mathrm{n}_{1} \\
\mathrm{n}_{2} \\
\mathrm{M} \\
\mathrm{n}_{\mathrm{k}}
\end{array}\right] \\
& \mathrm{N}_{\mathrm{t}+2 \mathrm{p}}=\mathrm{T} \cdot \mathrm{N}_{\mathrm{p}}+\mathrm{R}=\mathrm{T} \cdot \mathrm{T} \cdot \mathrm{N}_{\mathrm{t}}+\mathrm{R} \\
& \mathrm{N}_{\mathrm{t}+2 \mathrm{p}}=\mathrm{T}^{2} \cdot \mathrm{N}_{\mathrm{t}}+\mathrm{R}
\end{aligned}
$$

Sendo: $\mathrm{N}_{\mathrm{t}}=$ vetor do número de árvores em classes diamétricas no ano $\mathrm{t} ; \mathrm{N}_{\mathrm{t}+\mathrm{p}}=$ vetor do número de árvores no final do período de crescimento $\mathrm{p} ; \mathrm{N}_{\mathrm{t}+2 \mathrm{p}}=$ vetor com a projeção do número de árvores em classes diamétricas no ano $\mathrm{t}+2 \mathrm{p}$; $\mathrm{O}$ subscrito $\mathrm{t}$ refere-se ao período inicial, $\mathrm{p}$ ao final do primeiro período de crescimento, sendo $\mathrm{t}+2 \mathrm{p}$ o período de predição.

\section{Análise estatística e paramétrica}

A construção da matriz de transição foi precedida da AED em virtude de duas razões principais: 1) suspeita de possíveis erros de medição do CAP; 2) presença de outliers decorrentes dos processos de medição utilizados ou em conseqüência da variação biológica dos fatores de sítio como solo, espécie e outros. De todos esses erros, a posição de medida é a mais importante por variar em cada ocasião nos inventários comerciais e por ser fixa em remedições de parcelas permanentes.

Para o cálculo das medianas, os dados foram organizados em classes de área, dividindo-se a área de 576 ha em módulos de 4 ha, totalizando 144 unidades. Em cada módulo amostral foi calculada a mediana do diâmetro e área basal e de seus respectivos incrementos periódicos.

No modelo de USHER, a razão entre os elementos da distribuição, no período, é considerada 
constante. Essa constante é a taxa real de crescimento da população, simbolizada pelo escalar $\lambda_{1}$ (lamba índice um). $\mathrm{O}$ vetor de equilíbrio $\mathrm{V}_{\mathrm{d}}$ associado às taxas estáveis de crescimento da população contidas no vetor $\mathrm{L}_{\mathrm{d}}$, deve satisfazer à seguinte condição:

$$
\begin{aligned}
& \mathrm{T} \cdot \mathrm{V}_{\mathrm{d}}=\mathrm{L}_{\mathrm{d}} \cdot \mathrm{V}_{\mathrm{d}} \\
& \left(\mathrm{T}-\mathrm{L}_{\mathrm{d}} \cdot \mathrm{I}\right) \cdot \mathrm{V}_{\mathrm{d}}=0 \quad \therefore \quad \operatorname{det}\left(\mathrm{T}-\mathrm{L}_{\mathrm{d}} \cdot \mathrm{I}\right)=0
\end{aligned}
$$

Sendo: $\mathrm{I}$ =matriz identidade; $\mathrm{L}_{\mathrm{d}} \& \mathrm{~V}_{\mathrm{d}}$ são respectivamente o autovalor e o autovetor da matriz $\mathrm{T}$.

$\mathrm{O}$ cálculo dos autovalores $\mathrm{L}_{\mathrm{d}}$ e autovetores $\mathrm{V}_{\mathrm{d}}$ foram feitos com base na matriz de transição $\mathrm{T}$, utilizando-se o Software MatLab, por dispor de algoritmo que permite a determinação das raízes latentes dominantes mesmo para matrizes não-simétricas.

$\mathrm{O}$ valor de $\lambda_{1}$ corresponde à taxa real de crescimento da população em equilíbrio. A razão $\lambda=\lambda_{1} /\left|\lambda_{2}\right|$ fornece o fator de aceleração do ritmo de crescimento na direção da condição máxima de equilíbrio que é o estágio clímax.

A situação de equilíbrio é descrita pela raiz dominante latente $\lambda_{1}$ de $L_{d}$ e seus autovetores contidos em $V_{d}$, sendo esperado que $\lim _{t \rightarrow \infty} T^{n} V_{d}=L_{d} V_{d}$. A distribuição de equilíbrio expressa pelo vetor $\mathrm{V}_{\mathrm{d}}$ ocorrerá independentemente da distribuição inicial, e é provavelmente uma função das probabilidades de transição em $\mathrm{T}$.

Uma maneira de se determinar a taxa de convergência para o equilíbrio, é examinar a razão $\lambda=\lambda_{1} /\left|\lambda_{2}\right|$ entre a raiz dominante $\left(\lambda_{1}\right)$ e o módulo da segunda maior raiz $\lambda_{2}$ de $L_{d}$.

Para a matriz de transição, é esperado que os valores das raízes sejam maiores do que as raízes correspondentes das matrizes potências. Para a matriz de distribuição diamétrica de USHER, a raiz dominante latente é o maior valor $\lambda_{i}$ do vetor $L_{d}$. Dada a condição $A_{i}+B_{i}=1$, mesmo havendo perda de indivíduos da população por mortalidade durante o processo de projeção, ainda assim, a população não diminuirá e a raiz dominante $\left(\lambda_{1}\right)$ de $\mathrm{L}_{\mathrm{d}}$ será sempre maior ou igual a 1.

Se $\lambda_{1}=1$, o número de indivíduos em cada classe permanecerá constante no tempo e pode-se dizer que a população é estacionária. Se $\lambda_{1}>1$, a população está-se expandindo e se $\lambda_{1}<1$, a população pode estar em processo de desaceleração de crescimento. As distribuições diamétricas, em nível de espécie, são governadas por diferentes taxas de crescimento e mortalidade em diferentes partes da amplitude diamétrica dependendo da posição fitossociológica dos indivíduos.

Tem sido observado que sob as mais diversas condições de uso em florestas, a raiz dominante latente e seu vetor associado apresentam propriedades consistentes com a biologi a desses ecossistemas, pois, com a significância biológica de diferentes valores de $L_{d}$, pode ser esperado um vetor de equilíbrio $V_{d}$ com o passar do tempo.

\section{RESULTADOS E DISCUSSÃO}

Para uma melhor visualização das principais diferenças entre os incrementos periódicos por classes de DAP (cm) e possíveis outliers, construiu-se os box plot por espécie em 3 níveis: 1) dados sem- tratamento; 2) dados organização por classe de mediana e, 3) dados remanescentes após remoção de valores extremos com base em stem-and-leaf.

Observou-se que os potenciais outliers podem ser em conseqüência da diversidade biológica dos fatores de crescimento e competição característicos de uma floresta em estágio clímax, ou podem ser erros 
de medição. Tanto a variabilidade biológica quanto o erro de medição são comuns em levantamentos desse tipo, tornando-se difícil discriminar os erros de medição, pois alguns tipos de outliers são inerentes da população. Nesse caso, dispõe-se da possibilidade de identificar os outliers mais severos mediante a detecção das observações extremas reveladas pelo gráfico de tronco-e-folhas. Isso permitiu remover uma parte significativa dos prováveis erros de medição, mantendo-se os demais outliers na análise.

De um modo geral, observou-se uma redução nítida na amplitude dos incrementos periódicos em conseqüência dos tratamentos dos dados. Tratando os dados com o uso da mediana, obteve-se uma redução significativa no número de observações extremas. Fazendo uso do gráfico de tronco-e-folhas, conseguiam-se melhorar os dados em 2 níveis: i) redução da dispersão dos incrementos periódicos e, ii) redução do número de pontos extremos.

De acordo com a Figura 2(a), os valores extremos estão presentes em quase todas as classes, exceto a classe [80-90). A comparação por contraste entre as Figuras 2(a) e 2(b) permite inferir que a simples ordenação dos incrementos em classes de área com base na mediana, foi suficiente para promover o equilíbrio da variância dos dados. Após a remoção dos valores extremos em destaque na Figura 2(c), obteve -se uma redução na amplitude dos dados de 37 para $14 \mathrm{~cm}$, equivalente a $65 \%$. Mas, a redu ção foi mais significativa em relação ao total de valores extremos, sendo possível observar que, de 7 classes, apenas 2 continuam apresentando outliers de natureza biológica.

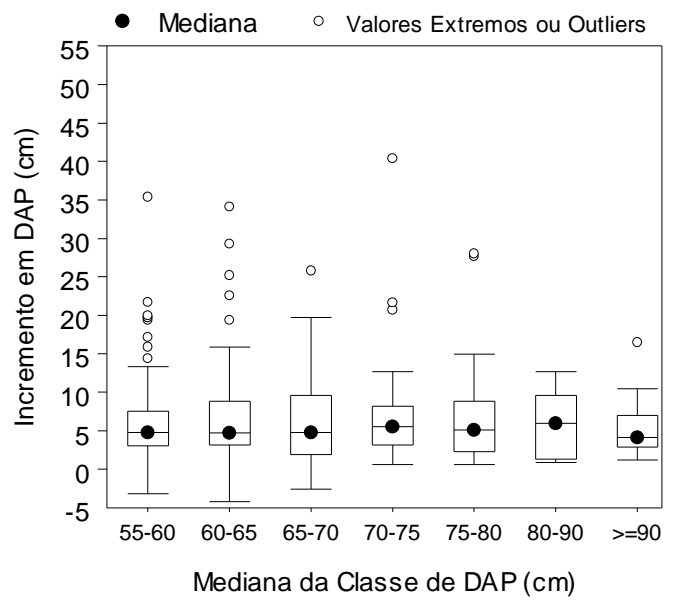

a

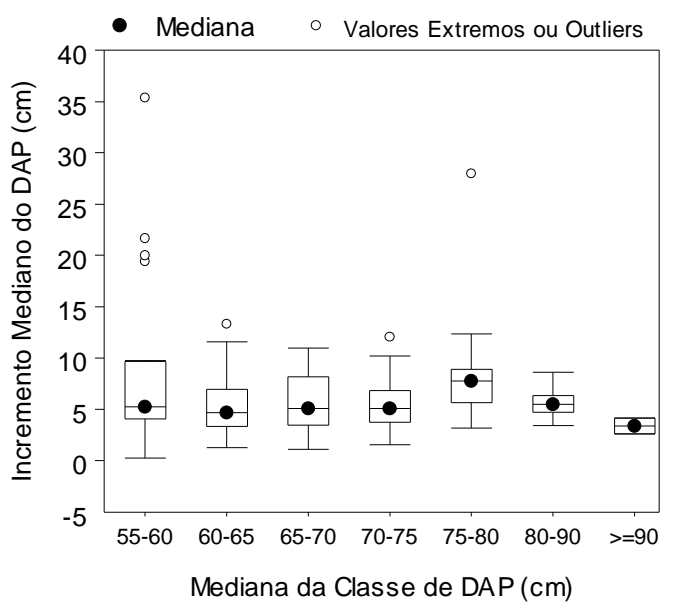

b

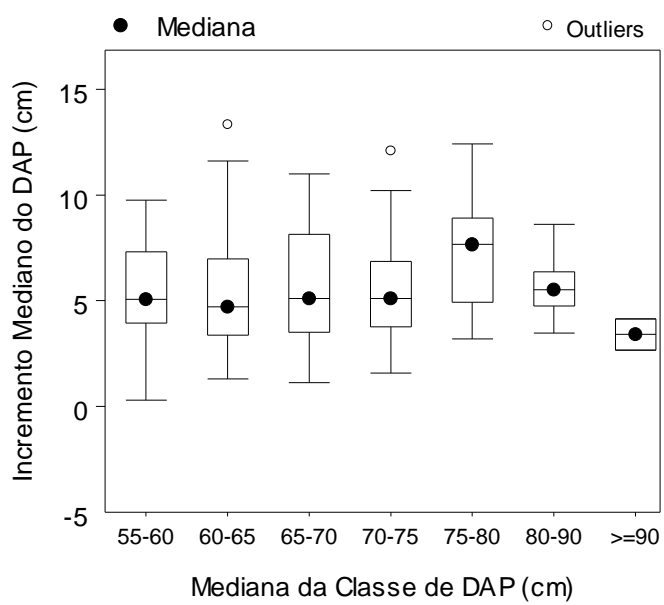

c

FIGURA 2: Box plot do incremento periódico por classe de DAP $(\mathrm{cm})$ para a Carapa guianensis Aubl. (Andiroba), sendo (a) dados sem-tratamento, (b) dados organizados por classe de mediana e (c) dados após remoção de valores extremos com base em stem-and-leaf. 
As observações discrepantes, constantes na Figura 3(a), mostram que os possíveis erros de medição resultantes dos incrementos anormais estão presentes em todas as classes, mesmo nas classes menores que são mais fáceis de serem medidas, o que se deve a erro sistemático ou aleatório. A maior variância, em termos de incremento periódico, ocorreu na classe [95-120).

Recorrendo-se ao efeito de contraste entre as Figuras 3(a) e 3(b), observa-se o quanto a ordenação dos dados, por classe de mediana, proporcionou uma melhor estabilidade na variância dos dados, resultado de grande importância para processos de modelagem. A Figura 3(c) mostra que, al ém da redução de $65 \%$ na variação da amplitude dos dados, com a remoção dos possíveis erros de medição apenas três classes [55-65), [65-75) e [85-95) de um total de seis, mostram ser portadoras de informações que puderam ser caracterizadas como outliers biológicos.

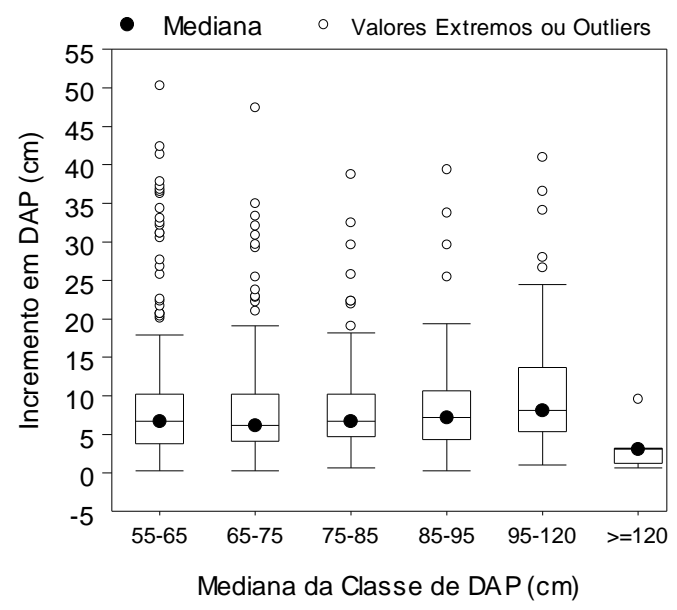

a

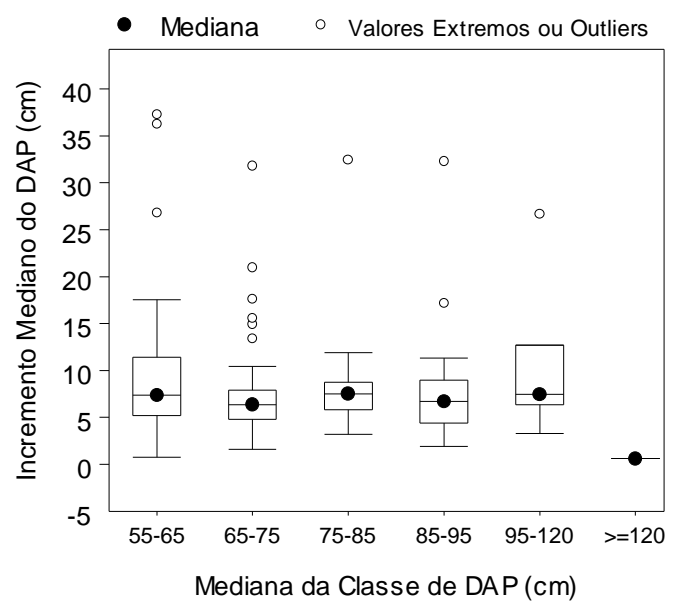

b

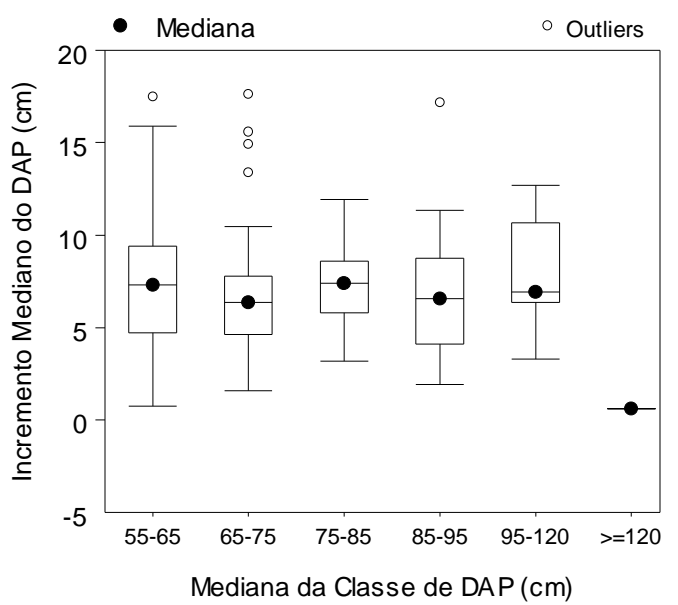

c

FIGURA 3: Box plot do incremento periódico por classe de DAP (cm) para a Manilkara huberi Ducke (Maçaranduba), sendo (a) dados sem-tratamento, (b) dados organizados por classe de mediana e (c) dados após remoção de valores extremos com base em stem-and-leaf.

De acordo com a Figura 4(a), no período 1984-2000, a forma da distribuição que, em 1984, apresentava assimetria positiva, em 2000, convergiu, assumindo a forma próxima da distribuição normal. Para 2016, espera-se que a Acariquara continue evoluindo com tendência para assimetria negativa e valor modal na classe [65-75).

A Figura 4(b) mostra que a Andiroba, em 1984, apresentava o perfil da distribuição diamétrica com 
valor modal na classe [60-65). A movimentação das classes mostra que, em 2000, a classe [65-70) foi a que registrou a menor alteração. A projeção para 2016 indica que, mantidas as taxas de crescimento observadas no período (1984-2000), a distribuição das árvores vivas deverá assumir a forma assimétrica negativa com o valor modal em destaque na classe [80-90).

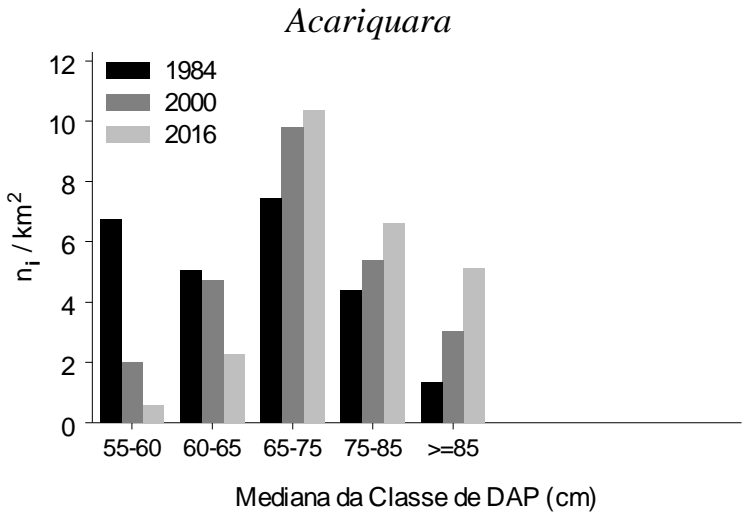

(a)

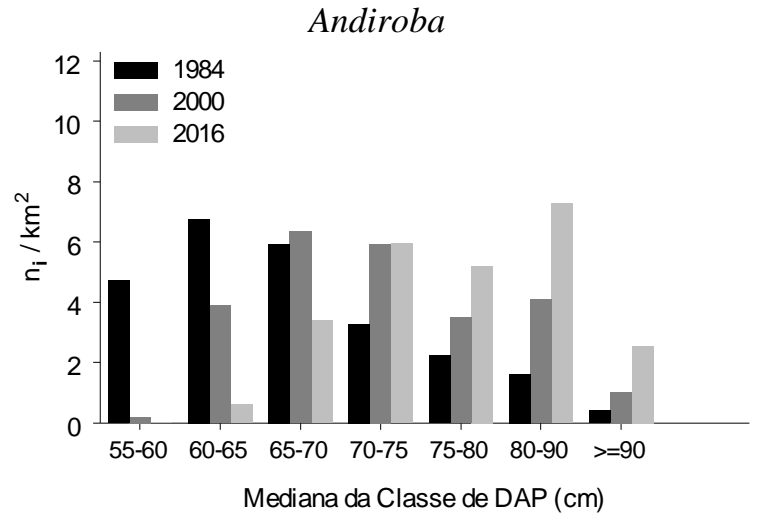

(b)

FIGURA 4: Evolução do DAP no período (1984-2000) e projeção por matriz de transição da abundância $\left(\mathrm{n}_{\mathrm{i}} / \mathrm{km}^{2}\right)$ em classes de DAP para 2016 - (b) Minquartia macrophylla Ducke (Acariquara) e Carapa guianensis Aubl. (Andiroba).

Observando-se a Figura 5(a), verifica-se, com base na evolução apresentada em 1984-2000, que a Castanheira, em 2016, deverá manter a forma da distribuição diamétrica estável, em conformidade com os níveis de 2000, incluindo o valor modal da classe [105-115).

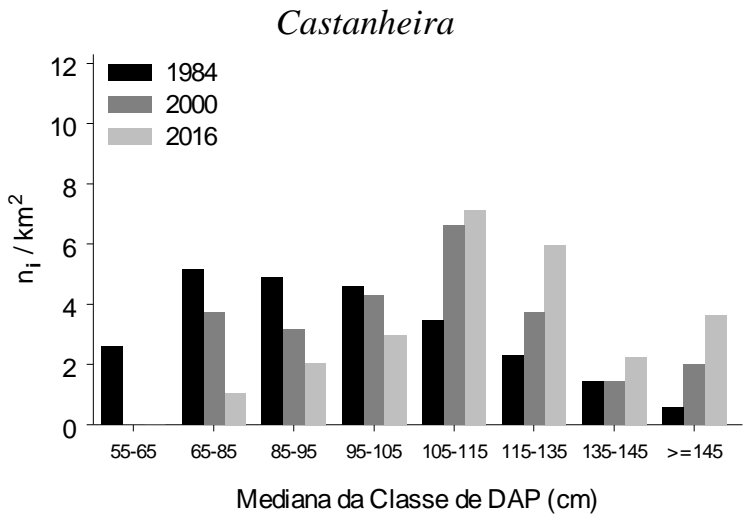

(a)

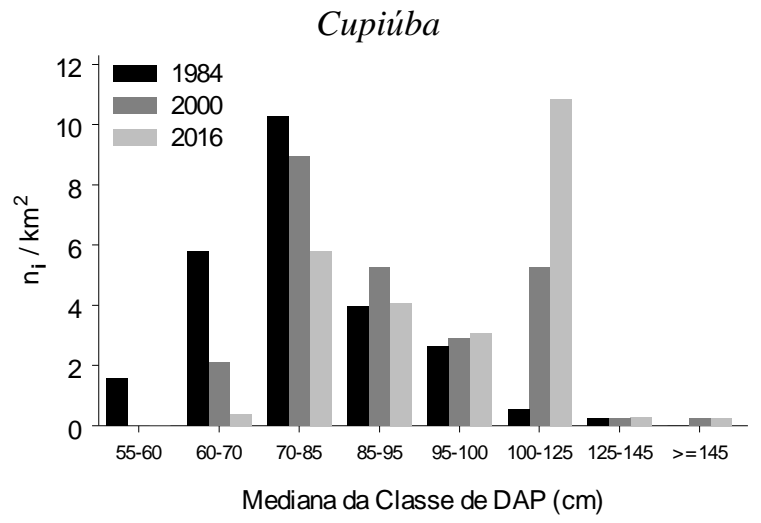

(b)

FIGURA 5: Evolução do DAP no período (1984-2000) e projeção por matriz de transição da abundância $\left(\mathrm{n}_{\mathrm{i}} / \mathrm{km}^{2}\right)$ em classes de DAP para 2016 - (a) Bertholletia excelsa Humb e Bonpl. (Castanheira) e (b) Goupia glabra Aubl. (Cupiúba).

Observa-se, pela Figura 5(b), que a Cupiúba, no período 1984-2000, apresentou uma transição evolutiva intra e interclasses sem-grandes alterações, mantendo a forma básica da distribuição. No entanto, para 2016, espera-se nívéis de mudanças mais acentuadas apenas na classe [100-125).

A Figura 6(a) indica, de acordo com a evolução projetada para 2016, que a Maçaranduba deverá mudar em termos de assimetria e valor modal, com tendência da assimetria tornar-se negativa e do valor modal deslocar-se da classe [75-85) para a classe [85-95). 
A Figura 6(b) mostra que a Tatajuba é o exemplo de uma espécie comercial com distribuição diamétrica bastante irregular e com a presença de intervalos assíncronos em termos de continuidade. $\mathrm{O}$ esperado é que em 2016, o seu perfil diamétrico mantenha a forma e o equilíbrio que a caracterizava em 2000.

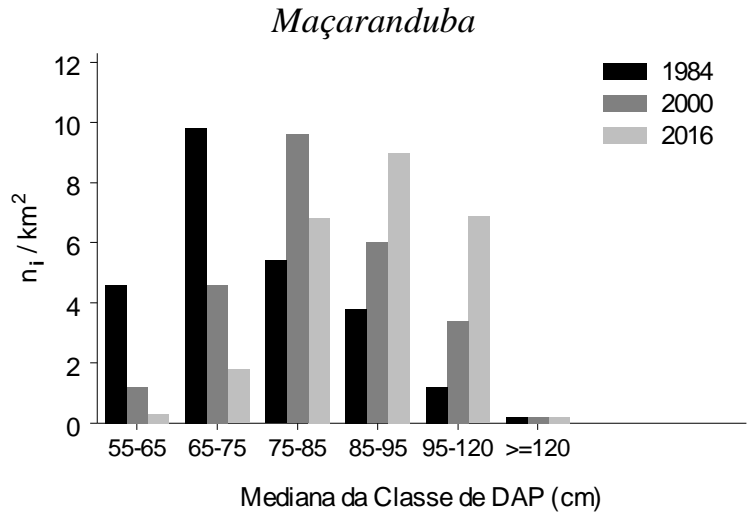

(a)

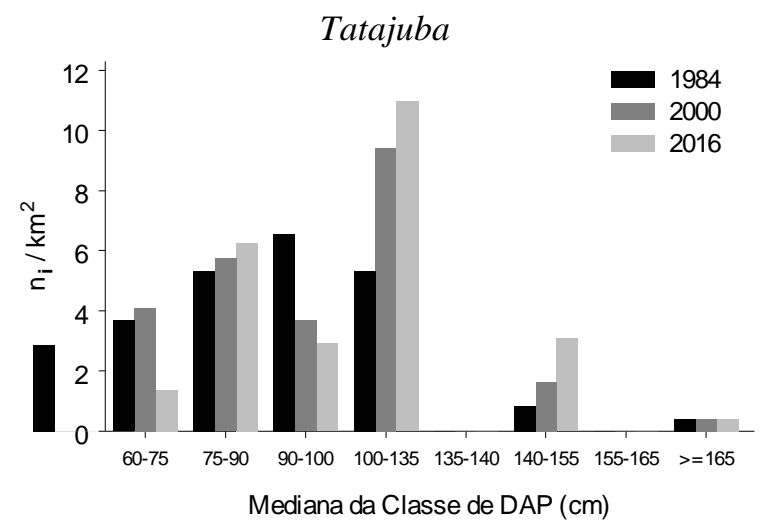

(b)

FIGURA 6: Evolução do DAP no período (1984-2000) e projeção por matriz de transição da abundância $\left(\mathrm{n}_{\mathrm{i}} / \mathrm{km}^{2}\right)$ em classes de DAP para 2016 - (a) Manilkara huberi Ducke (Maçaranduba) e (b) Bagassa guianensis Aubl. (Tatajuba).

Nas Tabelas 1 e 2, estão armazenados os resultados das projeções por matriz de transição das duas espécies de maior frequência com 93,23 e 82,47\%, ou seja, Acariquaqara e Maçaranduba respectivamente.

TABELA 1: Evolução do DAP no período (1984-2000) e projeção da abundância $\left(\mathrm{n}_{\mathrm{i}} / \mathrm{km}^{2}\right)$ em classes de DAP por matriz de probabilidades de transição desenvolvida para Carapa guianensis Aubl. (Andiroba).

\begin{tabular}{|c|c|c|c|c|c|c|c|c|c|c|}
\hline \multirow{2}{*}{$\begin{array}{c}\mathrm{CD}_{\text {med }}(\mathrm{cm}) \\
2000\end{array}$} & \multicolumn{7}{|c|}{ Mediana da classe de DAP $\left(\mathrm{CD}_{\text {med }}\right)-\mathrm{cm}, 1984$} & \multicolumn{3}{|c|}{ Abundância $\left(\mathrm{n}_{\mathrm{i}} / \mathrm{km}^{2}\right)$} \\
\hline & {$[55-60)$} & {$[60-65)$} & {$[65-70)$} & {$[70-75)$} & {$[75-80)$} & {$[80-90)$} & $>=90$ & 1984 & 2000 & 2016 \\
\hline$[55-60)$ & 0,04 & & & & & & & 4,7 & 0,2 & 0,0 \\
\hline$[60-65)$ & 0,65 & 0,12 & & & & & & 6,8 & 3,9 & 0,6 \\
\hline$[65-70)$ & 0,30 & 0,58 & 0,17 & & & & & 5,9 & 6,4 & 3,4 \\
\hline$[70-75)$ & & 0,30 & 0,52 & 0,25 & & & & 3,3 & 5,9 & 6,0 \\
\hline$[75-80)$ & & & 0,24 & 0,56 & 0,09 & & & 2,3 & 3,5 & 5,2 \\
\hline$[80-90)$ & & & 0,07 & 0,19 & 0,91 & 0,63 & & 1,6 & 4,1 & 7,3 \\
\hline$>=90$ & & & & & & 0,38 & 1,00 & 0,4 & 1,0 & 2,6 \\
\hline
\end{tabular}

TABELA 2: Evolução do DAP no período (1984-2000) e projeção da abundância $\left(\mathrm{n}_{\mathrm{i}} / \mathrm{km}^{2}\right)$ em classes de DAP por matriz de probabilidades de transição desenvolvida para Manilkara huberi Ducke (Maçaranduba).

\begin{tabular}{|c|c|c|c|c|c|c|c|c|c|}
\hline \multirow{2}{*}{$\begin{array}{c}\mathrm{CD}_{\text {med }}(\mathrm{cm}) \\
2000\end{array}$} & \multicolumn{6}{|c|}{ Mediana da classe de DAP $\left(\mathrm{CD}_{\text {med }}\right)-\mathrm{cm}, 1984$} & \multicolumn{3}{|c|}{ Abundância $\left(\mathrm{n}_{\mathrm{i}} / \mathrm{km}^{2}\right)$} \\
\hline & {$[55-65)$} & {$[65-75)$} & {$[75-85)$} & [85-95) & {$[95-120)$} & $>=120$ & 1984 & 2000 & 2016 \\
\hline [55-65) & 0,26 & & & & & & 4,6 & 1,2 & 0,3 \\
\hline$[65-75)$ & 0,39 & 0,29 & & & & & 9,8 & 4,6 & 1,8 \\
\hline$[75-85)$ & 0,30 & 0,63 & 0,37 & & & & 5,4 & 9,6 & 6,8 \\
\hline$[85-95)$ & 0,04 & 0,08 & 0,63 & 0,42 & & & 3,8 & 6,0 & 9,0 \\
\hline$[95-120)$ & & & & 0,58 & 1,00 & & 1,2 & 3,4 & 6,9 \\
\hline$>=120$ & & & & & & 1,00 & 0,2 & 0,2 & 0,2 \\
\hline
\end{tabular}


A Tabela 1 mostra que a probabilidade $\mathrm{a}_{\mathrm{i}}$ de uma árvore permanecer na mesma classe cresce progressivamente de 0,04 na classe [55-60) para 0,25 na classe [70-75), decresce em níveis mais acentuados na classe [75-80), assumindo um valor de 0,09 e volta a aumentar em níveis significativos, atingindo 0,63 na classe [80-90) e estabilizando-se em 1,00 na última classe [>=90. Enquanto isso, a probabilidade $b_{i}$ de uma árvore mover-se para classes seguintes decresce de 0,65 na classe [55-60) até 0,52 na classe [65-70), é crescente nos intervalos [70-75) e [75-80) com 0,57 e 0,91 respectivamente e decrescente com 0,38 em [8090).

Na Tabela 2, a probabilidade $\mathrm{a}_{\mathrm{i}}$ de uma árvore permanecer na mesma classe mantém-se crescente, variando de 0,26 na classe [55-65) até 1,00 nas classes [95-120) e [ $>=120$. Por outro lado, a probabilidade $\mathrm{b}_{\mathrm{i}}$ de uma árvore mover-se para classe posteriores aumenta de 0,39 na classe [55-65) para 0,63 nas classes [65-75) e [75-85), em seguida, decresce para 0,58 na classe [85-95).

TABELA 3: Taxas de crescimento por espécie com base nos autovalores da matriz de transição (T) com $\lambda_{1}>\left|\lambda_{2}\right|$.

\begin{tabular}{l|l|c|c}
\hline Espécie & \multicolumn{1}{c|}{ Hábito } & $\lambda_{1}$ & $\lambda=\lambda_{1} /\left|\lambda_{2}\right|$ \\
\hline Maçaranduba & Clímax de crescimento lento-tolerante à sombra & 1,00 & 1,00 \\
Tatajuba & Pioneira & 1,00 & 1,00 \\
Acariquara & Clímax de crescimento lento-tolerante à sombra & 1,00 & 1,38 \\
Andiroba & Clímax de crescimento rápido-demandante de luz & 1,00 & 1,60 \\
Castanheira & Clímax de crescimento rápido-demandante de luz & 1,00 & 2,00 \\
Cupiúba & Pioneira & 1,00 & 2,05 \\
\hline
\end{tabular}

Segundo Wadsworth (1977), o valor de $\lambda_{1}$, próximo de 1 , sugere que não está ocorrendo mudanças no número total de árvores da população. Um valor de $\lambda_{1}=1,2$ indica uma taxa de crescimento rápida do número de árvores, enquanto a distribuição proporcional, dentro das classes diamétricas, permanece constante.

As taxas de crescimento da Tabela 3 permitem estabelecer uma analogia com os resultados dos grupos ecológicos definidos por EMBRAPA (2000) cuja classificação em espécies clímax de crescimento lento, clímax de crescimento rápido e pioneiras pôde ser aferida com base nos valores de $\lambda_{1} \mathrm{e} \lambda$.

A análise dos autovalores da matriz de transição (T), constantes na Tabela 3, revelam que o valor de $\lambda_{1}$ foi igual a 1 para todas as espécies, indicando que a população dessas espécies é estacionária e mantém-se em equilíbrio. As taxas de convergência para o estágio clímax, representado por $\lambda$, mostra que entre as espécies classificadas como clímax de crescimento lento a Maçaranduba está em total equilíbrio, pois o valor de $\lambda$ é igual a 1 e que a Acariquara com $\lambda=1,38$ está-se movendo em um ritmo muito mais lento na direção do estágio clímax do que as demais espécies. Das espécies consideradas clímax de rápido crescimento, a Castanheira com $\lambda=2,00$ apresenta uma taxa de crescimento $40 \%$ maior do que a Andiroba cujo valor de $\lambda$ foi de 1,60. Dentre as espécies pioneiras, a Tatajuba mostrou taxas de crescimento constante em ambas as situações e para a Cupiúba, o valor de $\lambda=2,05$ é um parâmetro indicativo que confirma a sua característica de espécie pioneira.

\section{CONCLUSÃO}

A análise da estrutura diamétrica mostrou que a evolução do DAP observada no período 1984-2000, apresentou comportamento diferenciado por espécie, embora mantendo a forma básica da distribuição típica de cada espécie, enquanto a projeção da estrutura diamétrica para 2016, usando matriz de transição, manteve-se com a tendência esperada, com base nas taxas de crescimento expressas pelas ra ízes latentes da matriz de transição, o que demonstra estar compatível com o perfil da estrutura diamétrica observada em 2000. 


\section{REFERÊNCIAS BIBLIOGRÁFICAS}

ADLARD, P. G. Procedures for monitoring tree growth and site change. Oxford: Forestry Institute, 1990.188p. (Tropical Forestry Paper, 23).

ALDER, D. GHAFOSIM: a projection system for natural forest growth and yield in Ghana. Consultancy Report to UK/ODA, 1990. 114p. (Unpublished).

. Growth modelling for mixed tropical forests. Oxford: Forestry Institute, 1995. 231 p. (Tropical Forestry Paper, 30).

ALDER, D.; SYNNOTT, T. J. Permanent sample plot techniques for mixed tropical forest. Oxford: Forestry Institute, 1992. 124 p. (Tropical Forestry Paper, 25).

ASSMANN, E. The principles of forest yield study. Oxford: Pergamon Press, 1970. 506p.

BARNETT, V.; LEWIS, T. Outliers in statistical data. Chichester: John Wiley, 1995. 584p.

DRAPER, N. R.; SMITH, H. Applied regression analysis. 2. ed. New York: John Wiley, 1981. 709p.

ELLISON, A. M. Exploratory data analysis and graphic display: design and analysis of ecological experiments. New York: Chapman \& Hall, 1993, p.14-41.

EMBRAPA. Relatório do workshop de implementação do projeto Dendrogene: conservação genética em florestas manejadas na Amazônia. Belém, 2000. 88p.

FUNDAÇÃO DE PESQUISAS FLORESTAIS DO PARANÁ. Inventário comercial da quadra de exploração n. 4 da Floresta Nacional do Tapajós. Curitiba, 1984. 429p.

GNANADESIKAN, R. Methods for statistical data analysis of multivariate observations. New Jersey: John Wiley, 1997. 353p.

HIGUCHI, N. Short-term growth of an undisturbed tropical moist forest in the Brazilian Amazon. 1987. 129p. Tese (Doutorado) - Michigan State University, Michigan, EUA.

HOAGLIN, D. C.; MOSTELLER, F.; TUKEY, J.W. Análise exploratória de dados: técnicas robustas-um guia. Salamandra, Almada, Portugal, 1992. 446p.

IBAMA. PNUD. Plano de manejo florestal da Floresta Nacional do Tapajós, Pará, Brasil. Belém, 1997. 109p.

LESLIE, P. H. On the use of matrices in certain population mathematics. Biometrika, v. 33, p. 183-212, 1945.

MOSTELLER, F.; TUKEY, J. W. Data analysis and regression : a second course in statistics. Reading, UK, Addison-wesley publishing company, 1997. 588p.

RADAMBRASIL. Projeto RADAMBRASIL. Rio de Janeiro: Departamento Nacional da Produção Mineral, 1976. (Levantamento de Recursos Naturais, 10, Folha AS 21).

SANQUETTA, C. R.; NIEFER, I. A.; VOLPI, N. M. P.; MOROKAWA, T. Análise de sensibilidade e risco em prognose volumétrica de florestas com matriz de transição. Agrárias. Curitiba, v. 16, n.1/2, p. 47-62, 1997.

SILVA, R.C.V. M. Identificação de espécimes botânicos. Belém, 2000. 31p.

STATGRAPHICS. User guide. Disponivel em: 〈http://www.statgraphics.com>. Acesso em: 1999.

TAYLOR, H. M.; KARLIN, S. An introduction to stochastic modeling. 3 ed. San Diego, California: Academic Press, 1998. 631p.

USHER, M. B. A matrix approach to the management of renewable resources, with special reference to selection forests-two extensions. J. Appl. Ecol., v. 3, p. 355-367, 1966.

USHER, M. B. A matrix model for forest management. Biometrics, v. 25, p. 309-315, 1969.

WADSWORTH, R. K. A study of diameter distributions of an uneven aged tropical forest by means of a transition matrix model. 1977. Thesis (Doctor of Philosophy) - College of Forest Resources, University of Washington, Washington, EUA.

NATIONAL INSTITUTE OF STANDARDS AND TECNOLOGY-NIST. Statistics for Scientists \& Engineers: exploratory data analysis. Disponivel em: 〈http://www.itl.nist.gov>. Acesso em: 1999. 